\title{
Bayesian Inference of the Evolution of a Phenotype Distribution on a Phylogenetic Tree
}

\author{
M. Azim Ansari* and Xavier Didelot ${ }^{\dagger, 1}$ \\ * Oxford Martin School, University of Oxford, Oxford OX1 3BD, United Kingdom, and ${ }^{\dagger}$ Department of Infectious Disease \\ Epidemiology, Imperial College London, London W2 1PG, United Kingdom
}

\begin{abstract}
The distribution of a phenotype on a phylogenetic tree is often a quantity of interest. Many phenotypes have imperfect heritability, so that a measurement of the phenotype for an individual can be thought of as a single realization from the phenotype distribution of that individual. If all individuals in a phylogeny had the same phenotype distribution, measured phenotypes would be randomly distributed on the tree leaves. This is, however, often not the case, implying that the phenotype distribution evolves over time. Here we propose a new model based on this principle of evolving phenotype distribution on the branches of a phylogeny, which is different from ancestral state reconstruction where the phenotype itself is assumed to evolve. We develop an efficient Bayesian inference method to estimate the parameters of our model and to test the evidence for changes in the phenotype distribution. We use multiple simulated data sets to show that our algorithm has good sensitivity and specificity properties. Since our method identifies branches on the tree on which the phenotype distribution has changed, it is able to break down a tree into components for which this distribution is unique and constant. We present two applications of our method, one investigating the association between HIV genetic variation and human leukocyte antigen and the other studying host range distribution in a lineage of Salmonella enterica, and we discuss many other potential applications.
\end{abstract}

KEYWORDS changepoint model; inheritability; phenotypic plasticity; phylogenetic tree; trait evolution

NDERSTANDING phenotypic variations and their relative association with genotypic variations is one of the central aims of molecular biology. The expression of a phenotype is usually dependent on both genetic and environmental factors, with heritability measuring their relative importance (Visscher et al. 2008). When the heritability is nonzero, genetically similar individuals are more likely to have similar phenotypes, and this is especially relevant for species that reproduce clonally, so that closely related individuals are virtually identical genetically. However, genotypephenotype maps are usually complex and phenotypic plasticity

Copyright (C) 2016 Ansari and Didelot

doi: 10.1534/genetics.116.190496

Manuscript received April 18, 2016; accepted for publication July 7, 2016; published Early Online July 11, 2016.

Available freely online through the author-supported open access option.

This is an open-access article distributed under the terms of the Creative Commons Attribution 4.0 International License (http://creativecommons.org/licenses/by/4.0/), which permits unrestricted use, distribution, and reproduction in any medium, provided the original work is properly cited.

Supplemental material is available online at www.genetics.org/lookup/suppl/doi:10. 1534/genetics.116.190496/-/DC1.

${ }^{1}$ Corresponding author: Department of Infectious Disease Epidemiology, Imperial

College London, Norfolk PI., London W2 1PG, United Kingdom. E-mail: xavier.

didelot@gmail.com means that phenotype expression can differ even for genetically identical individuals due to dependency on environmental factors (DeWitt et al. 1998; Agrawal 2001). Conversely, observing closely related individuals with the same phenotype does not necessarily imply a low importance of environmental factors, since close relatives are also likely to live in the same environmental conditions (Visscher et al. 2008). The same effect also occurs in sexually reproducing species as evolutionary forces such as spatial population structure, environmental pressures, and inbreeding result in groups within which individuals are more genetically homologous, and therefore more phenotypically similar, than individuals from different groups (Pritchard et al. 2000; Lawson et al. 2012).

To understand the relationship between a phenotype and a genotype, it is necessary to investigate how the phenotype is distributed according to genotypic values. This requires quantifying how the genotypes are related to each other, which is often achieved using phylogenetic trees (Yang and Rannala 2012). For clonal organisms, the tree may represent the clonal genealogy of how individuals are related with one another for nonrecombinant regions (Didelot and Falush 
2007; Didelot et al. 2010). For sexual organisms, the phylogenies may be built for individual genomic loci, resulting in so-called gene trees by contrast with the species tree that contains them (Maddison 1997). Visual inspection of a phylogenetic tree with tips annotated by phenotypes gives a first impression of their relationship, and this type of figure features heavily in the molecular biology literature of both clonal and sexual organisms. A more quantitative approach is, however, needed if the tree is too large to be shown, if the interesting patterns are too subtle to be seen, or to estimate evolutionary parameters and test competing hypotheses.

Phylogenetic comparative methods can be used, for example to test the phylogenetic signal in a phenotype (Hillis and Huelsenbeck 1992; Blomberg et al. 2003) or to compare the association between two phenotypes given the phylogeny (Garland et al. 2005), but do not provide a complete description of the phenotype distribution on a tree. One of the most popular phylogenetic comparative methods is ancestral state reconstruction of the phenotype given the tree (Cunningham et al. 1998; Pagel 1999). Application of this method can provide quantitative insights, for example an estimate of the phenotypic evolutionary rate. The maximum-likelihood approach to ancestral state reconstruction (Yang et al. 1995) has been extended in many ways by refining the model of phenotypic evolution on the tree, for example by allowing the detection of branches where the phenotypic evolutionary rate changes (Revell 2008; Revell et al. 2011). However, ancestral state reconstruction is problematic for any phenotype with imperfect heritability: Identical genotypes can then have different phenotypic values, implying an infinitely high rate of phenotypic evolution between them that is not biologically meaningful. Other difficulties arise if the phylogeny is imperfectly reconstructed or the phenotype inaccurately measured, which is always a possibility. Consequently, ancestral state reconstruction does not always provide reliable results, for example when applied to phylogeography (De Maio et al. 2015)

When heritability is not complete, a phenotypic measurement can be seen as just one realization from the phenotypic distribution of a given individual, with this distribution being what evolves on the tree rather than the phenotypic measurement itself. Based on this idea, here we present a novel Bayesian statistical method that takes as input a phylogenetic tree and discrete tip phenotype measurements and identifies the branches on which the phenotype distribution has changed. The tree is therefore divided into monophyletic and paraphyletic groups that have unique distributions over the phenotype space. We also perform Bayesian hypothesis testing (Kass and Raftery 1995) to assess whether there is evidence for different parts of the tree having distinct phenotype distributions. We build a stochastic model in which changepoints occur on a phylogenetic tree (Didelot et al. 2009), each of which affects the distribution of observed phenotype for the descendent leaves. Careful parameterization enables the use of a fixed-dimension Markov chain Monte Carlo (MCMC) algorithm (Gilks et al. 1995) to sample from the posterior distribution of the model parameters, and we reserve reversible jumps (Green 1995) to compare the model with a model without any changepoint. In the following sections we present our model, our inference procedure, and the results of simulation studies to measure the sensitivity and specificity of our method. Finally we present the application of our method to two real data sets in human immunodeficiency virus (HIV) evasion and bacterial ecology.

\section{Model and Methods}

\section{Description of the model}

We consider that changepoints happen as a Poisson process with rate $\lambda$ on the branches of the input tree. For a phenotype with $K$ categories, we model each changepoint event as a new probability mass function $\mathbf{q}=\left(q_{1}, \ldots, q_{K}\right)$ that specifies the probability of having each of the $K$ phenotypes for the individuals affected by the changepoint. Figure 1 illustrates the model for $K=2$. The observed phenotype of each individual is shown on the tips of the tree, which are colored black and red. Changepoints have happened on three branches that divided the tree into four sections (white, blue, green, and yellow). All individuals in the same section have the same distribution $\mathbf{q}$ over the phenotype space.

Let $N$ and $B$ denote the number of tips and branches in the tree, respectively (if the tree is bifurcating, then $B=2 N-2$ ). We define $\mathbf{b}=\left(b_{1}, \ldots, b_{B}\right)$ as a binary vector with $B$ elements that represent the branches of the tree. If branch $i$ holds at least one changepoint, then $b_{i}=1$; otherwise $b_{i}=0$. Let $m$ denote the number of sections of the tree divided according to $\mathbf{b}$ (Figure 1). The likelihood of the observed phenotypes of the individuals $D$ is given by

$$
p\left(D \mid \mathbf{q}_{1}, \ldots, \mathbf{q}_{m}, \mathbf{b}\right)=\prod_{j=1}^{K} q_{1 j}^{x_{1 j}} \ldots \prod_{j=1}^{K} q_{m j}^{x_{m j}},
$$

where $\mathbf{q}_{i}=\left(q_{i 1}, \ldots, q_{i k}\right)$ and $q_{i j}$ gives the probability that an individual in section $i$ expresses phenotype $j$, so that $\sum_{j=1}^{K} q_{i j}=1$ for $i=1, \ldots, m$. We also define $\mathbf{x}_{i}=\left(x_{i 1}, \ldots, x_{i K}\right)$, where $x_{i j}$ is the number of observed individuals in section $i$ that have expressed phenotype $j$, so that $\sum_{i=1}^{m} \sum_{j=1}^{K} x_{i j}=N$.

Assuming that the length of branch $i$ is known, the prior probabilities of branch $i$ having no or at least one changepoint are respectively $\operatorname{Pr}\left(b_{i}=0 \mid \lambda\right)=e^{-\lambda l_{i}}$ and $\operatorname{Pr}\left(b_{i}=1 \mid \lambda\right)=$ $1-e^{-\lambda l_{i}}$, so that

$$
\operatorname{Pr}(\mathbf{b} \mid \lambda)=\prod_{i=1}^{B}\left(e^{-\lambda l_{i}}\right)^{1-b_{i}}\left(1-e^{-\lambda l_{i}}\right)^{b_{i}} .
$$

We consider a flat Dirichlet prior for all $\mathbf{q}_{i}$ such that $p\left(\mathbf{q}_{i}\right)=\Gamma(K)$ and an exponential prior on $\lambda$ with mean $1 / T$, where $T=\sum_{i=1}^{B} l_{i}$ is the sum of the branch lengths of the tree. This implies a parsimonious prior expectation of one for the number of changepoints on the tree. 


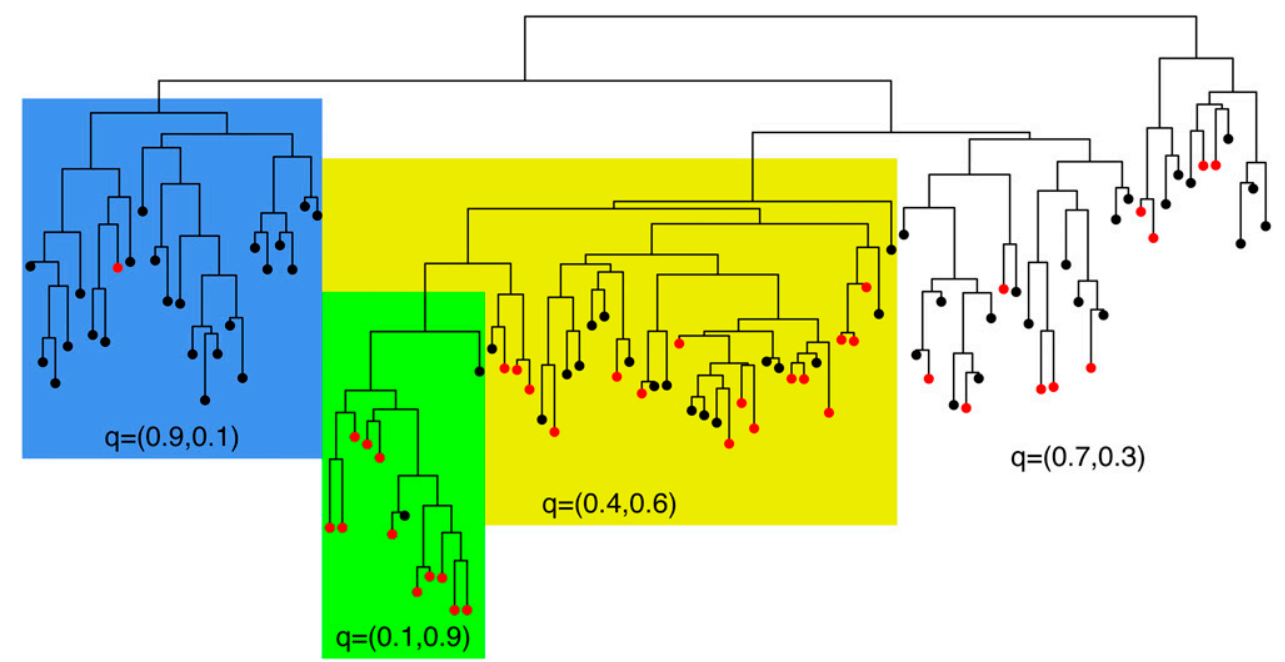

Figure 1 Illustration of the model. Changepoints occurred on three branches, which divided the tree into four sections (white, blue, green, and yellow), each of which has different probabilities of the first (black) and second (red) phenotypes.

We are now in a position to describe the posterior distribution of the model parameters $\mathbf{q}_{i}, \ldots, \mathbf{q}_{m}, \mathbf{b}$ and $\lambda$ :

$$
\begin{aligned}
p\left(\mathbf{q}_{1}, \ldots, \mathbf{q}_{m}, \mathbf{b}, \lambda \mid D\right) & =p\left(D \mid \mathbf{q}_{1}, \ldots, \mathbf{q}_{m}, \mathbf{b}, \lambda\right) p\left(\mathbf{q}_{1}, \ldots, \mathbf{q}_{m}, \mathbf{b}, \lambda\right) / p(D) \\
& \propto p\left(D \mid \mathbf{q}_{1}, \ldots, \mathbf{q}_{m}, \mathbf{b}\right) p\left(\mathbf{q}_{1}\right) \ldots p\left(\mathbf{q}_{m}\right) p(\mathbf{b} \mid \lambda) p(\lambda) \\
& \propto(\Gamma(K))^{m} \prod_{i=1}^{m} \prod_{j=1}^{K} q_{i j}^{x_{i j}} \prod_{s=1}^{B}\left(e^{-\lambda l_{s}}\right)^{1-b_{s}}\left(1-e^{-\lambda l_{s}}\right)^{b_{s}} T e^{-T \lambda} .
\end{aligned}
$$

The dimensionality of the model parameters changes with $\mathbf{b}$ as the number of sections on the tree depends on $\mathbf{b}$ and each section has its own distribution over the phenotype space. This could potentially be addressed using reversible jumps (Green 1995). Instead we marginalize all the $\mathbf{q}_{i}$, which results in a fixed-dimension model. The marginal posterior density for $\mathbf{b}$ and $\lambda$ is given by

$$
\begin{aligned}
p(\mathbf{b}, \lambda \mid D)= & \int_{\mathbf{q}_{1}} \cdots \int_{\mathbf{q}_{m}} p\left(D \mid \mathbf{q}_{1}, \ldots, \mathbf{q}_{m}, \mathbf{b}\right) \\
& \times p\left(\mathbf{q}_{1}\right) \cdots p\left(\mathbf{q}_{m}\right) d \mathbf{q}_{1} \cdots d \mathbf{q}_{m} p(\mathbf{b} \mid \lambda) p(\lambda) / p(D) \\
& \propto(\Gamma(K))^{m} \prod_{i=1}^{m} \prod_{j=1}^{K} \int_{0}^{1} q_{i j}^{x_{i j}} d q_{i j} T e^{-T \lambda} \prod_{s=1}^{B}\left(e^{-\lambda l_{s}}\right)^{1-b_{s}}\left(1-e^{-\lambda l_{s}}\right)^{b_{s}} \\
& \propto(\Gamma(K))^{m} \prod_{i=1}^{m} \frac{\prod_{j=1}^{K} \Gamma\left(x_{i j}+1\right)}{\Gamma\left(K+\sum_{j=1}^{K} x_{i j}\right)} T e^{-T \lambda} \prod_{s=1}^{B}\left(e^{-\lambda l_{s}}\right)^{1-b_{s}}\left(1-e^{-\lambda l_{s}}\right)^{b_{s}} .
\end{aligned}
$$

\section{Inference}

We use a MCMC (Gilks et al. 1995) to sample from the posterior distribution of $\mathbf{b}$ and $\lambda$. We use a symmetric proposal for $\mathbf{b}$ where the proposed value $\mathbf{b}^{\star}$ is the same $\mathbf{a s} \mathbf{b}$ except for one randomly chosen branch $i$ for which $b_{i}^{\star}=1-b_{i}$. Therefore if the randomly chosen branch $i$ holds a changepoint in $\mathbf{b}$, it does not hold a changepoint in $\mathbf{b}^{\star}$ and vice versa. To update $\lambda$ we propose from a normal density with mean equal to the current value of $\lambda$ and variance equal to 0.1 ; i.e., $\lambda^{\star} \mid \lambda \sim \mathcal{N}(\lambda, 0.1)$. When the proposed $\lambda^{\star}$ is lower than zero, the move is rejected and the chain stays at $\lambda$. The calculation of the Metropolis-
Hastings acceptance ratios is given in Supplemental Material, File S1.

\section{Model selection}

We want to assess whether there is any evidence for differential distribution of phenotypes on different parts of the tree. We compare our model (indexed 1) against the null model (indexed 0) of no changepoints on the tree, which is equivalent to $\lambda=0$, by calculating the Bayes factor (Kass and Raftery 1995) for the two models. To do this we use reversible-jump moves (Green 1995) to sample from the joint distribution $p\left(\left(j, \boldsymbol{\theta}_{j}\right) \mid D\right)$, where $j$ is the index of the model and $\boldsymbol{\theta}_{j}$ is the parameters of model $j$. For a move from null to alternative ( 0 to 1 ) model, to match dimensions we generate two random variables $u$ and $\mathbf{v}$ and map them such that $\left(\lambda^{\star}, \mathbf{b}^{\star}\right)=(u, \mathbf{v})$. In addition we set the proposal distribution for $u$ and $\mathbf{v}, q(u, \mathbf{v})$ in model 0 to be the same as the prior distribution on $\lambda$ and $\mathbf{b}$ in model 1 . Thus for a proposed move from model 0 to model 1 we have

$$
q(u, \mathbf{v})=q(u) q(\mathbf{v} \mid u)=T e^{-T u} \prod_{i=1}^{B}\left(e^{-u l_{i}}\right)^{1-v_{i}}\left(1-e^{-u l_{i}}\right)^{v_{i}} .
$$

The probability of acceptance of this move is given by

$$
\begin{aligned}
h\left((0) \rightarrow\left(1,\left(\lambda^{\star}, \mathbf{b}^{\star}\right)\right)\right) & =1 \wedge \frac{p\left(1,\left(\lambda^{\star}, \mathbf{b}^{\star}\right) \mid D\right) p(1 \rightarrow 0)}{p(0 \mid D) p(0 \rightarrow 1) q((u, \mathbf{v}) \mid 0)}\left|\frac{\partial\left(\lambda^{\star}, \mathbf{b}^{\star}\right)}{\partial(u, \mathbf{v})}\right| \\
& =1 \wedge \frac{p(1,(u, \mathbf{v}) \mid D) p(1 \rightarrow 0)}{p(0 \mid D) p(0 \rightarrow 1) q((u, \mathbf{v}) \mid 0)} \times 1 \\
& =1 \wedge \frac{p(D \mid(u, \mathbf{v}), 1) p((u, \mathbf{v}) \mid 1) p(1) p(1 \rightarrow 0)}{p(D \mid 0) p(0) p(0 \rightarrow 1) q((u, \mathbf{v}) \mid 0)} \\
& =1 \wedge \frac{p(D \mid(u, \mathbf{v}), 1) p(1 \rightarrow 0)}{p(D \mid 0) p(0 \rightarrow 1)}
\end{aligned}
$$

A move from model 1 with parameters $(\lambda, \mathbf{b})$ to model 0 is made deterministically and is accepted with probability 


$$
h((1,(\lambda, \mathbf{b})) \rightarrow(0))=1 \wedge \frac{p(D \mid 0) p(0 \rightarrow 1)}{p(D \mid(\lambda, \mathbf{b}), 1) p(1 \rightarrow 0)} .
$$

We set $p(1 \rightarrow 0)=0.05$ and $p(0 \rightarrow 1)=0.5$ and we assume the prior probabilities of the two models are equal, $p(0)=p(1)=0.5$.

\section{Simulation studies}

To investigate the performance of our method, we performed two simulation studies, each of which involved repetition over many simulated data sets. In all of these simulations for simplicity we used a binary phenotype and sampled from the posterior distribution of the model parameters, using $10^{7}$ iterations of our MCMC algorithm. All of these simulations were implemented for a single genealogy simulated using the coalescent model (Rosenberg and Nordborg 2002) with 1000 leaves shown in Figure S1. First, we tested how the number of individuals affected by a changepoint and the magnitude of the change in phenotype distribution affect the statistical power to detect a changepoint. Second, we tested the model selection procedure and the relationship between the posterior expectation of number of changepoints and the true numbers of changepoints. Third, we quantified the effect of threshold on the point estimate of $\mathbf{b}$.

\section{Data availability}

All the methods described in this article are implemented in a software package called TreeBreaker, which is freely available for download at https://github.com/ansariazim/ TreeBreaker.

\section{Results}

\section{Simulation study of statistical power}

This simulation study was designed to assess the power of the method to detect changepoints on the branches of the tree. The power depends on two factors: the magnitude of the change in the distribution over the phenotype categories, which we refer to as $p$, and the number of individuals affected by the changepoint, which we refer to as $n$. The probability of each phenotype is 0.5 before the changepoint, and after the changepoint the probability of one phenotype increases by $p$ whereas the probability of the other phenotype decreases by $p$. Changepoints with small $p$ are difficult to detect as they result in small changes to the observed pattern of distribution of phenotype that are likely to happen by chance alone. Changepoints with small $n$ are also difficult to distinguish as lack of data makes the inference more uncertain. We expect that changepoints with large $p$ and large $n$ are easier to detect.

The space of $n \times p$ was divided into a grid where $n=$ $(10,30,60,130,330,500)$ and $p=(0.1,0.2,0.3,0.4,0.5)$. For each node of the grid $\left(n_{i}, p_{j}\right), 50$ data sets were simulated where in each case an appropriate branch of the tree shown in Figure S1 was chosen to have a changepoint, with the remaining branches not having any changepoints. Figure 2 shows for each node of the grid the mean marginal posterior probability of having a changepoint for the branch with the changepoint. A changepoint that causes large changes to the distribution of the phenotype categories and affects a large number of individuals is inferred with a high posterior probability. Changepoints that cause small changes in the distribution or affect few individuals or both result in small posterior probability of having a changepoint.

\section{Simulation study of model and parameter inference}

This simulation study was designed to assess our model selection procedure, the effect of number of changepoints on the inference, and the effect of cutoff threshold on the point estimate of $\mathbf{b}$. We simulated 100 data sets for each case of $0,1, \ldots, 10$ changing branches in the tree. The distribution over the phenotypes was uniformly sampled in each case. For each simulated data set the Bayes factor of our model against the null model was estimated (Figure 3A). For the 100 data sets with no changepoint on the tree, all the estimated Bayes factors supported the null model (no changepoint on the tree). Changepoints that result in small changes in the distribution or affect a small number of individuals will not be detected. Therefore for some of data sets with a single changing branch there is no support for the alternative model, but for some there is strong support for the alternative model. As the number of changing branches on the tree increases, the number of data sets with posterior support for the alternative model increases. Overall, our method is conservative and should not result in significant evidence for the existence of changepoints unless there are substantial data to support it.

Next, we used the simulations to gauge the relationship between the true number of simulated changing branches and its posterior expectation, estimated using Bayesian model averaging (Hoeting et al. 1999). Figure 3B illustrates the results. In the absence of any changepoint, the mean of posterior expectation of number of changing branches is always close to zero. When there are changing branches on the tree, the posterior expectation is downward biased compared to the real value. This is expected as our method cannot detect a changepoint that results in small changes in the distribution or affects few individuals or both. As a result our method is conservative in estimating the number of changepoints on the tree.

In addition we used the simulation results to assess the effect of a cutoff threshold on the point estimate of $\mathbf{b}$. For each of the data sets we inferred a point estimate for $\mathbf{b}$ by applying a threshold to the consensus representation of $\mathbf{b}$ (marginal posterior probability of having a changepoint for each branch of the tree). The threshold was changed from 0 to 1 with increments of 0.01 . For each threshold value, the false positive rate and the true positive rate across all of our 1100 simulations were calculated. Figure 3C shows the true positive rate as a function of the false positive rate. This so-called receiver operating characteristic (ROC) curve has a high area under the curve of 0.891, indicative of good performance of the algorithm (Bradley 1997). The choice of the cutoff 


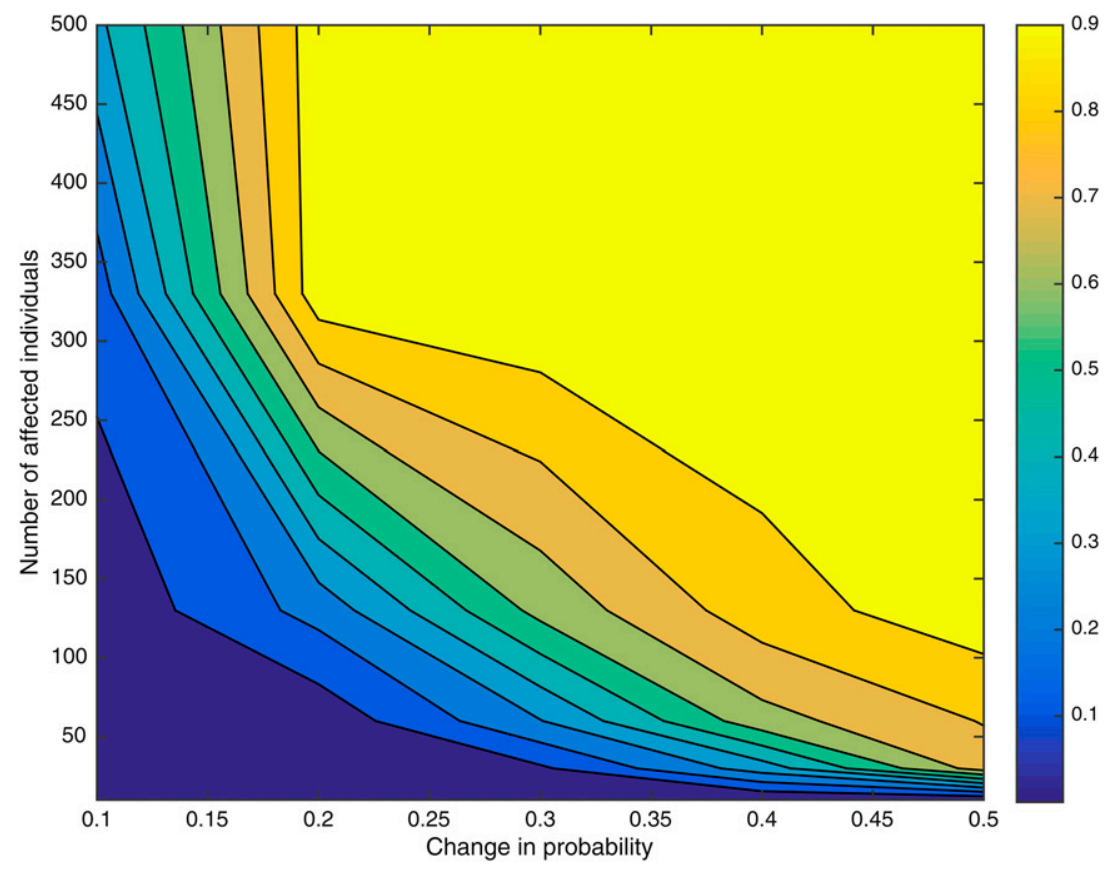

Figure 2 Relationship between statistical power and the number of affected individuals by a changepoint and the magnitude of the change in phenotype distribution. Shown is a contour plot of the mean posterior probability of having a changepoint as a function of number of affected individuals $(n)$ and the magnitude of the change in distribution ( $p$ ). The space of $n \times p$ was divided into a grid where $n=(10,30,60,130,330,500)$ and $p=(0.1,0.2,0.3,0.4,0.5)$. threshold is a trade-off between minimizing the number of incorrectly inferred changepoints and maximizing the number of correctly inferred changepoints. This choice depends on the application and the weight given to sensitivity and specificity in the application.

To test the sensitivity of our method to inaccuracies in the input phylogenetic tree, we repeated the runs for the same simulated data sets described above but using for inference a tree that had been randomized as follows. Patristic distances were computed for all pairs of leaves in the original tree, each pairwise distance $d$ was replaced with a uniform random draw from interval $[0.1 d ; 1.9 d]$, and a new tree was computed from these new distances, using UPGMA. Both model selection (Figure S2A) and the inference of the number of changepoints to have happened on the tree (Figure S2B) remained accurate in spite of the randomization of the tree, which shows that our inference method is relatively robust to small inaccuracies in the phylogenetic reconstruction.

\section{Detecting cytotoxic T-lymphocytes escape mutations in HIV}

Human leukocyte antigen (HLA) type I genes encode proteins that are present on the surface of almost all human cells. When a cell is infected with a virus, the viral protein is cleaved and small segments of it called epitopes are presented on the cell surface by the HLA-encoded proteins. These proteins have a certain amount of affinity and thus in people with the same HLA allele, the same epitope will be recognized and presented on the cell surface. Cytotoxic T lymphocytes (CTLS) are part of the adaptive immune response and recognize these epitopes before destroying the infected cell. A mutation in one of these epitopes can result in no or weak binding of the peptide to the HLA-encoded protein or result in lack of recognition by the T-cell receptor. Such mutations lead to the virus escaping the immune response of the host. As these mutations can have a fitness cost on transmission to a host with different HLA repertoire, they may revert back to the wild type (Leslie et al. 2004). Thus the escape mutations on the virus genome are correlated with the host's HLA alleles.

However, to detect these associations one has to account for the possible geographical structuring that could be present in the data. For instance, the distribution of HIV subtypes is different in various parts of the world and HLA allele profiles are also distinct in different populations across the world. When sampling is across different countries or ethnic groups, it is possible that HLA alleles will be associated with specific clusters of the virus simply because of geographical structuring. Several methods have been suggested to account for the nonrandom distribution of HLA alleles on the tips of the phylogenetic tree (Bhattacharya et al. 2007; Carlson et al. $2008,2012)$. We propose that using our algorithm, one can determine whether host HLA alleles are randomly distributed on the tips of the virus phylogenetic tree or whether there are clades where the distributions are distinct from each other. The result can then be used to perform stratified association studies conditioned on the clades with distinct HLA distribution.

We used previously published data (Rousseau et al. 2008) on a cohort of 261 South Africans to detect HLA-driven evolution of HIV. In this study whole-genome viral sequences were aligned and then divided into 10 fragments of $1000 \mathrm{nu}-$ cleotides overlapping by 50 nucleotides. Each partition was then used to produce a maximum-likelihood phylogenetic tree. The HLA alleles of the patients were also typed. We used the 10 phylogenetic trees from this data set and the HLA information of the patients as the inputs to our algorithm, considering the presence and absence of each HLA allele separately. This resulted in 1197 runs of our software. Figure S3 shows the histogram of the Bayes factors estimated by 
A

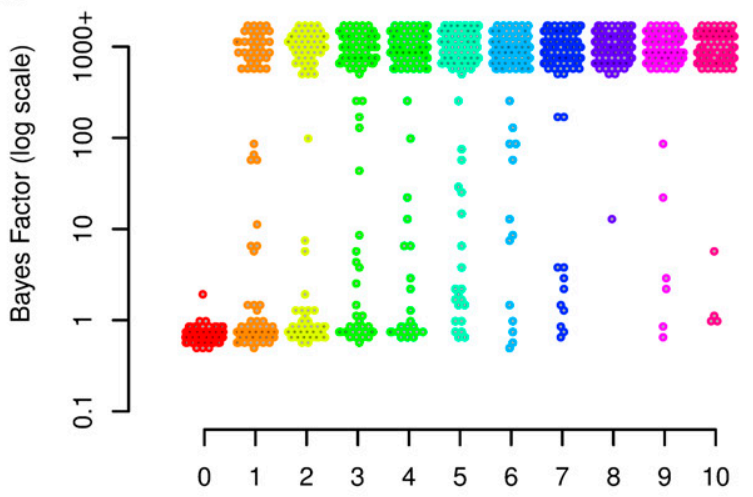

B

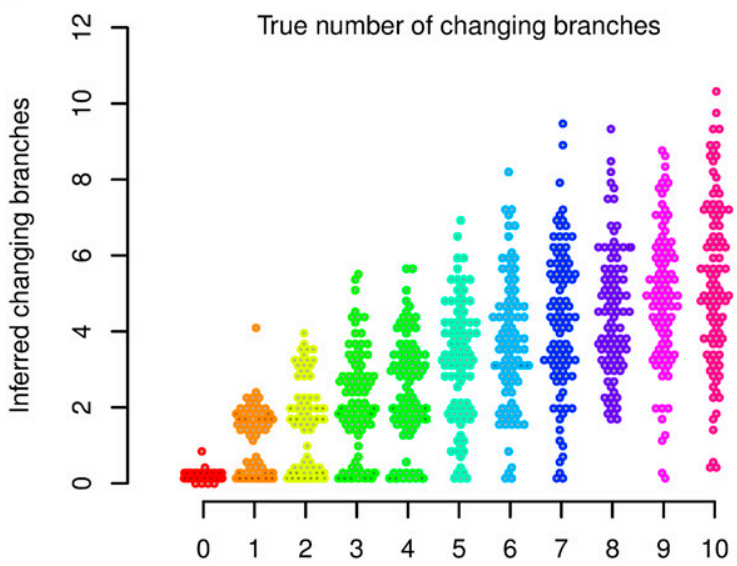

C

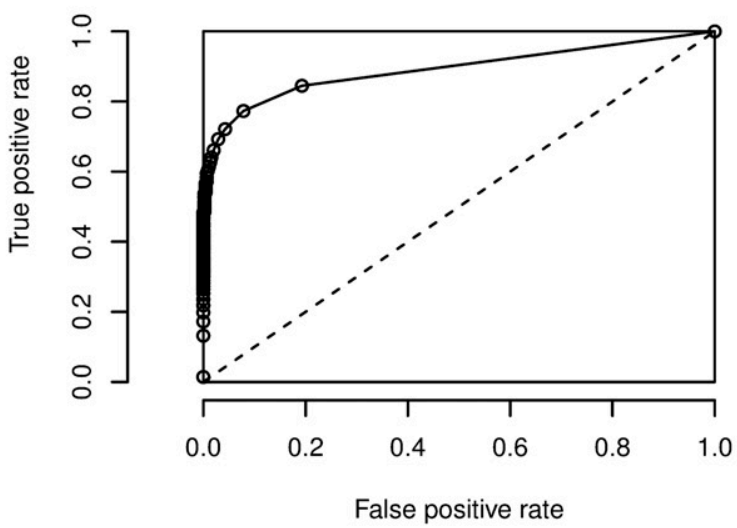

Figure 3 Simulation study of model and parameter inference. (A) Bayes factor values for the changepoint model vs. the null model, as a function of the number of changing branches used in the simulation. (B) Distribution of posterior mean number of changing branches as a function of the true number of simulated changing branches. (C) ROC curve: true positive rate as a function of the false positive rate.

each run. Only the distribution of HLA allele B57 on the tree of the first region of the HIV genome had a Bayes factor conclusively rejecting the null model of no association (Figure 4). There is a clade of 12 viral individuals where 10 of the hosts have the B57 allele, whereas across the rest of the tree there are only 7 other hosts with B57 alleles. This clear nonrandom distribution of the HLA allele B57 could be due to transmission of the virus between closely related people. However, we do not detect the same association between the other 9 trees from the rest of the genome and HLA allele B57. An alternative explanation may be that HLA allele B57 has a significant effect on the evolution of the first 1000 nucleotides of the virus, since HLA allele B57 is associated with slow progression to disease following HIV infection (Altfeld et al. 2003; Miura et al. 2009).

\section{Inferring host range within a lineage of Salmonella enterica}

Salmonella enterica is a bacterial pathogen made of multiple lineages with different host ranges (Uzzau et al. 2000; Didelot et al. 2011; Achtman et al. 2012). Many lineages can infect a wide range of animals, whereas some are mostly found in specific hosts and yet others have become restricted to a single host type, for example the Typhi and Paratyphi A lineages that evolved in convergence toward infecting only humans (Didelot et al. 2007). The Typhimurium DT104 lineage has been responsible for a global multidrug-resistant epidemic since the 1990s in both humans and farm animals (Glynn et al. 1998; Mølbak et al. 1999; Threlfall 2000). Typhimurium DT104 can infect both animals and humans, but it is unclear whether there are sublineages within DT104 that infect one host type more than the other and to what extent the epidemics in animals and humans are associated. Traditional molecular typing techniques do not provide enough genetic resolution to answer this question. A recent study sequenced the whole genomes of 142 human strains and 120 animal strains isolated in Scotland between 1990 and 2011 (Mather et al. 2013). A maximum-likelihood tree was computed based on the nonrecombinant core genome, using RAxML (Stamatakis 2006), and the phenotype was taken to be animal vs. human source of isolation at the tips of the tree. We applied ancestral state reconstruction to this data set, using maximum likelihood under an equal-rates model (Figure S4). A high rate of state change was estimated, corresponding to an expected total of 981 changes throughout the tree. This rate was driven by short branches separating human and animal isolates. Consequently, longer branches were expected to contain several changes, and the uncertainty about the state at ancestral nodes was high, with a nearly 50/50 probability for the animal and human states (Figure S4).

We then applied our own algorithm to this tree, to analyze the evolution of the phenotype distribution over the phylogenetic tree. The null model of random distribution of hosts around the tree was decisively rejected in favor of the changepoint model, with the reversible-jump MCMC never exploring the null model after initial burn-in. The posterior mean number of changing branches was 9.7 , with the $95 \%$ credibility interval ranging from 5 to 16 . Changes in the host range were especially evident on four branches (Figure 5), corresponding to posterior probabilities of $99 \%, 95 \%, 90 \%$, and $72 \%$, with two further branches with probability $54 \%$, one with $39 \%$, and all 




Figure 4 Application to HIV immunology. Shown is a phylogenetic tree of 261 HIV-infected individuals from the first 1000 nucleotides with the tips colored according to presence and absence of HLA allele B57 in the host. The thickness and color of the branches are proportional to the posterior probability of having a changepoint.

others $<20 \%$. Among the 4 branches with highest support, the oldest corresponds to an increase in the frequency of infection of animals for a large clade of 265 isolates within DT104. The other 3 branches all occurred within this clade and correspond to three separate further increases in the frequency of infection of animals for three subclades containing 12,15 , and 59 isolates, respectively. These results confirm and refine the original conclusions of the study in which the data were presented (Mather et al. 2013), that the epidemic of
DT104 in Scotland was not homogenous in humans and animals. Specifically, a sublineage increasingly became restricted to infecting only animals and not humans, which could be the result of either adaptation or niche segregation.

\section{Discussion}

This study is based on the concept of phenotype distribution, which is the distribution of phenotypes that a given genotype 


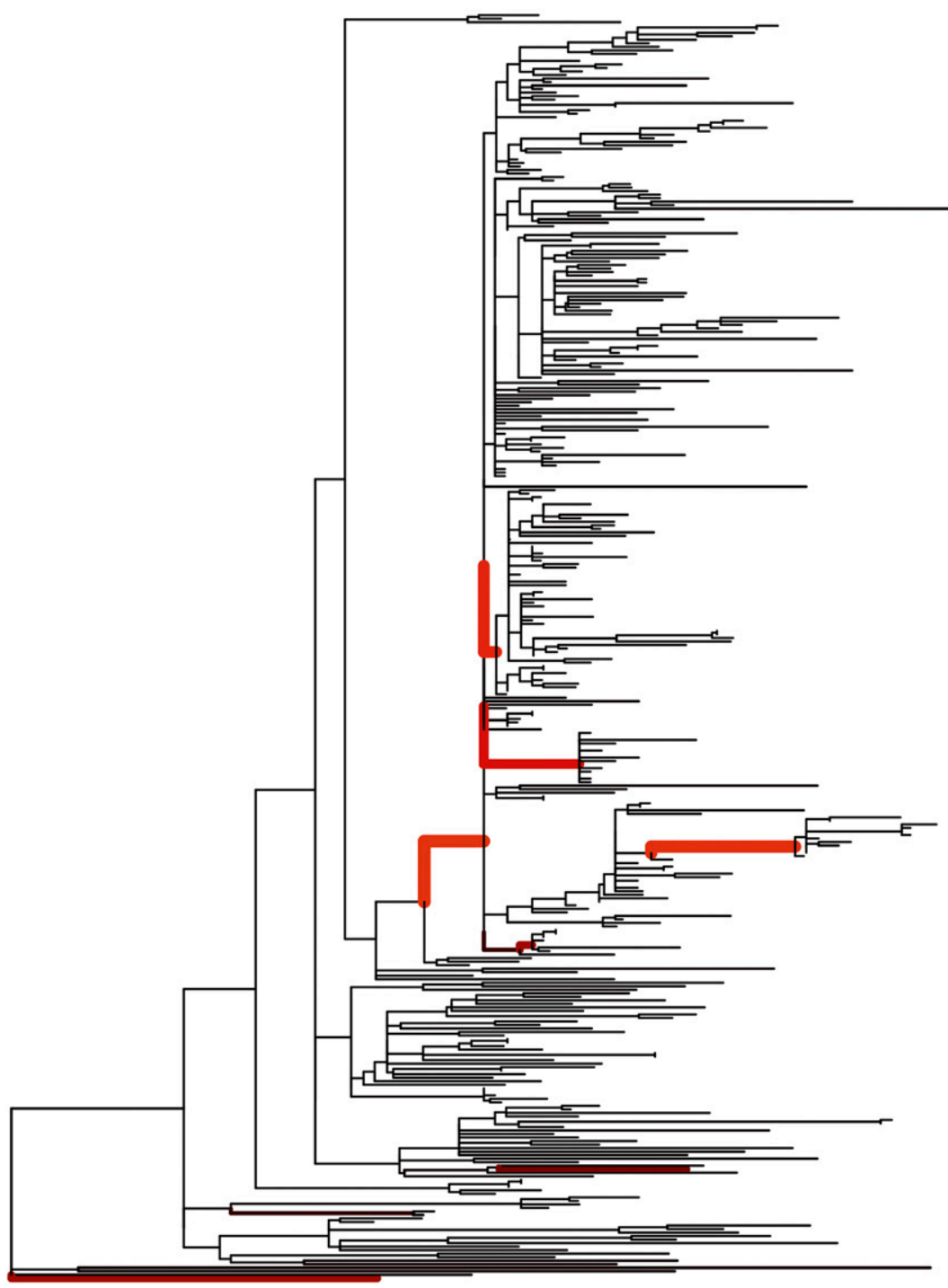

Figure 5 Application to Salmonella ecology. Shown is a maximum-likelihood phylogenetic tree from a previous study of Typhimurium DT104 (Mather et al. 2013), with the color on the right indicating the isolates came from either human (red) or animal (black) sources. The results of our algorithm are shown by the thickness and redness of the branches, which are both proportional to the posterior probability of host range change on the given branch.

may express depending on environmental factors, as a result of phenotypic plasticity (DeWitt et al. 1998; Agrawal 2001). We presented a model in which the phenotype distribution is allowed to change along the branches of a phylogenetic tree and an efficient Bayesian method to perform inference under this model. Our model for the evolution of the phenotype distribution is related to previous work on trait evolution with intraspecific variability, where the trait values recorded at the tips of a tree are drawn from a distribution whose parameters evolved as a Brownian motion on the branches of the tree (Felsenstein 2008; Revell and Reynolds 2012; Kostikova et al. 2016). A key difference, however, is that here we considered that there are parts of the tree in which the underlying parameters remain constant, so that identifying these component parts becomes the main objective of inference. We showed using both simulated and real data that given phenotype observations for the leaves of a phylogeny, our method can detect branches on which the phenotype distribution changed significantly. Consequently, a phylogeny can be demarcated into lineages with distinct phenotype distributions.
There are many ways in which our approach could be extended, for example to be applicable to continuous rather than categorical phenotype measurements (Hadfield and Nakagawa 2010; Felsenstein 2012) or to allow the evolution of the phenotype distribution to be more progressive, for example by making the distribution after a changepoint to be correlated with, rather than independent from, the distribution before the changepoint. In our examples, the trees were estimated using maximum likelihood and therefore the branch lengths measure sequence distance rather than time. The relationship between such branch lengths and phenotypic evolution is still an open question (Cunningham et al. 1998). We did not attempt to model the potential for error in either the input phylogeny or the input phenotype measurements. Uncertainty about the tree could be accounted for by applying our method to a sample of trees from the posterior distribution of the trees that are produced by Bayesian phylogenetic software such as MrBayes and BEAST (Huelsenbeck and Ronquist 2001; Drummond et al. 2012). However, we expect that a little inaccuracy in the tree would 
not drastically affect the result of our method and likewise for the phenotype measurement, because the results depend on phenotype distributions that are themselves stochastic. This is unlike methods that consider changes in the phenotype itself, such as ancestral state reconstructions (Yang et al. 1995), for which a mistake in a single phenotype measurement implies an additional evolutionary event for the phenotype. When considering phenotypes with imperfect heritability (Visscher et al. 2008), we argue that modeling the evolution of the phenotype distribution is more biologically relevant than modeling the evolution of the phenotype measurement.

There are many research areas in which the method we proposed could be useful, and we presented two examples in HIV immunology and bacterial ecology. For example, our approach could help provide a definition of microbial species. Detecting incipient speciation requires distinguishing between ecologically distinct populations in the same community (Ferris et al. 2003; Sikorski and Nevo 2005; Johnson et al. 2006). In this case the phenotype would be ecological or pathogenicity measurements, and the aim is to determine whether different phylogenetic clades have distinct distributions over the measurable ecological quantities (Achtman and Wagner 2008; Fraser et al. 2009). Another potential area of application is genome-wide association studies (GWAS) in organisms that reproduce clonally. Population structure is a confounding effect in GWAS (Marchini et al. 2004) and this is especially important for clonal organisms (Earle et al. 2016). One way to account for this population structure would be to use our method to find the clades on the phylogenetic tree where the phenotype of interest is uniquely distributed and perform GWAS stratified by those clusters.

\section{Acknowledgments}

The authors thank Remi Bardenet, Daniel Falush, Philip Maybank, and Gil McVean for their insightful discussions. This research was made possible by a James Martin fellowship (awarded to M.A.A.). X.D. received funding from the Biotechnology and Biological Sciences Research Council (BB/L023458/1) and the National Institute for Health Research (HPRU-2012-10080).

\section{Literature Cited}

Achtman, M., and M. Wagner, 2008 Microbial diversity and the genetic nature of microbial species. Nat. Rev. Microbiol. 6: 431-440.

Achtman, M., J. Wain, F.-X. Weill, S. Nair, Z. Zhou et al., 2012 Multilocus sequence typing as a replacement for serotyping in Salmonella enterica. PLoS Pathog. 8: e1002776.

Agrawal, A. A., 2001 Phenotypic plasticity in the interactions and evolution of species. Science 294: 321-326.

Altfeld, M., M. M. Addo, E. S. Rosenberg, F. M. Hecht, P. K. Lee et al., 2003 Influence of HLA-B57 on clinical presentation and viral control during acute HIV-1 infection. AIDS 17: 2581-2591.

Bhattacharya, T., M. Daniels, D. Heckerman, B. Foley, N. Frahm et al., 2007 Founder effects in the assessment of HIV polymorphisms and HLA allele associations. Science 315: 1583-1586.

Blomberg, S. P., T. Garland, and A. R. Ives, 2003 Testing for phylogenetic signal in comparative data: behavioral traits are more labile. Evolution 57: 717-745.

Bradley, A. P., 1997 The use of the area under the ROC curve in the evaluation of machine learning algorithms. Pattern Recognit. 30: 1145-1159.

Carlson, J. M., Z. L. Brumme, C. M. Rousseau, C. J. Brumme, P. Matthews et al., 2008 Phylogenetic dependency networks: inferring patterns of CTL escape and codon covariation in HIV-1 Gag. PLoS Comput. Biol. 4: e1000225.

Carlson, J. M., J. Listgarten, N. Pfeifer, V. Tan, C. Kadie et al., 2012 Widespread impact of HLA restriction on immune control and escape pathways of HIV-1. J. Virol. 86: 5230-5243.

Cunningham, C. W., K. E. Omland, and T. H. Oakley, 1998 Reconstructing ancestral character states. Trends Ecol. Evol. 5347: 361-366.

De Maio, N., C.-H. Wu, K. M. O'Reilly, and D. Wilson, 2015 New routes to phylogeography: a Bayesian structured coalescent approximation. PLoS Genet. 11: e1005421.

DeWitt, T. J., A. Sih, and D. S. Wilson, 1998 Cost and limits of phenotypic plasticity. Trends Ecol. Evol. 13: 77-81.

Didelot, X., and D. Falush, 2007 Inference of bacterial microevolution using multilocus sequence data. Genetics 175: 12511266.

Didelot, X., M. Achtman, J. Parkhill, N. R. Thomson, and D. Falush, 2007 A bimodal pattern of relatedness between the Salmonella Paratyphi A and Typhi genomes: Convergence or divergence by homologous recombination? Genome Res. 17: 61-68.

Didelot, X., A. Darling, and D. Falush, 2009 Inferring genomic flux in bacteria. Genome Res. 19: 306-317.

Didelot, X., D. Lawson, A. Darling, and D. Falush, 2010 Inference of homologous recombination in bacteria using whole-genome sequences. Genetics 186: 1435-1449.

Didelot, X., R. Bowden, T. Street, T. Golubchik, C. Spencer et al., 2011 Recombination and population structure in Salmonella enterica. PLoS Genet. 7: e1002191.

Drummond, A. J., M. A. Suchard, D. Xie, and A. Rambaut, 2012 Bayesian phylogenetics with BEAUti and the BEAST 1.7. Mol. Biol. Evol. 29: 1969-1973.

Earle, S. G., C.-H. Wu, J. Charlesworth, N. Stoesser, N. C. Gordon et al., 2016 Identifying lineage effects when controlling for population structure improves power in bacterial association studies. New Microbiol. 1: 16041.

Felsenstein, J., 2008 Comparative methods with sampling error and within-species variation: contrasts revisited and revised. Am. Nat. 171: 713-725.

Felsenstein, J., 2012 A comparative method for both discrete and continuous characters using the threshold model. Am. Nat. 179: $145-156$.

Ferris, M. J., M. Kühl, A. Wieland, and D. M. Ward, 2003 Cyanobacterial ecotypes in different optical microenvironments of a 68 degrees $\mathrm{C}$ hot spring mat community revealed by $16 \mathrm{~S}-23 \mathrm{~S}$ rRNA internal transcribed spacer region variation. Appl. Environ. Microbiol. 69: 2893-2898.

Fraser, C., E. J. Alm, M. F. Polz, B. G. Spratt, and W. P. Hanage, 2009 The bacterial species challenge: making sense of genetic and ecological diversity. Science 323: 741-746.

Garland, T., A. F. Bennett, and E. L. Rezende, 2005 Phylogenetic approaches in comparative physiology. J. Exp. Biol. 208: 30153035.

Gilks, W., S. Richardson, and D. Spiegelhalter, 1995 Markov Chain Monte Carlo in Practice. CRC Press, Cleveland, OH/Boca Raton, FL.

Glynn, M. K. A., C. H. Bopp, W. Dewitt, P. Dabney, M. Mokhtar et al., 1998 Typhimurium Dt104 infections in the United States. N. Engl. J. Med. 338: 1333-1338. 
Green, P. J., 1995 Reversible jump Markov chain Monte Carlo computation and Bayesian model determination. Biometrika 82: 711.

Hadfield, J., and S. Nakagawa, 2010 General quantitative genetic methods for comparative biology: phylogenies, taxonomies and multi-trait models for continuous and categorical characters. J. Evol. Biol. 23: 494-508.

Hillis, D. M., and J. P. Huelsenbeck, 1992 Signal, noise, and reliability in molecular phylogenetic analyses. J. Hered. 83: 189-195.

Hoeting, J. A., D. Madigan, A. E. Raftery, and C. T. Volinsky, 1999 Bayesian model averaging: a tutorial. Stat. Sci. 14: 382-401.

Huelsenbeck, J. P., and F. Ronquist, 2001 MRBAYES: Bayesian inference of phylogenetic trees. Bioinformatics 17: 754-755.

Johnson, Z. I., E. R. Zinser, A. Coe, N. P. McNulty, E. M. S. Woodward et al., 2006 Niche partitioning among Prochlorococcus ecotypes along ocean-scale environmental gradients. Science 311: 1737-1740.

Kass, R. E., and A. E. Raftery, 1995 Bayes factors. J. Am. Stat. Assoc. 90: 773.

Kostikova, A., D. Silvestro, P. B. Pearman, and N. Salamin, 2016 Bridging inter-and intraspecific trait evolution with a hierarchical Bayesian approach. Syst. Biol. 65: 417-431.

Lawson, D. J., G. Hellenthal, S. Myers, and D. Falush, 2012 Inference of population structure using dense haplotype data. PLoS Genet. 8: e1002453.

Leslie, A. J., K. J. Pfafferott, P. Chetty, R. Draenert, M. M. Addo et al., 2004 HIV evolution: CTL escape mutation and reversion after transmission. Nat. Med. 10: 282-289.

Maddison, W. P., 1997 Gene trees in species trees. Syst. Biol. 46: 523-536.

Marchini, J., L. R. Cardon, M. S. Phillips, and P. Donnelly, 2004 The effects of human population structure on large genetic association studies. Nat. Genet. 36: 512-517.

Mather, A. E., S. W. J. Reid, D. J. Maskell, J. Parkhill, M. C. Fookes et al., 2013 Distinguishable epidemics of multidrug-resistant Salmonella Typhimurium DT104 in different hosts. Science 341: 1514-1517.

Miura, T., M. A. Brockman, A. Schneidewind, M. Lobritz, F. Pereyra et al., 2009 HLA-B57/B*5801 human immunodeficiency virus type 1 elite controllers select for rare Gag variants associated with reduced viral replication capacity and strong cytotoxic T-lymphocyte recognition. J. Virol. 83: 2743-2755.

Mølbak, K., D. Baggesen, F. Møller Aarestrup, J. Ebbesen, J. Engberg et al., 1999 An outbreak of multidrug-resistant, quinolone-resistant Salmonella enterica serotype typhimurium DT104. N. Engl. J. Med. 341: 1420-1425.

Pagel, M., 1999 Inferring the historical patterns of biological evolution. Nature 401: 877-884.

Pritchard, J. K., M. Stephens, and P. Donnelly, 2000 Inference of population structure using multilocus genotype data. Genetics 155: 945-959.

Revell, L., 2008 On the analysis of evolutionary change along single branches in a phylogeny. Am. Nat. 172: 140-147.

Revell, L. J., and G. R. Reynolds, 2012 A new Bayesian method for fitting evolutionary models to comparative data with intraspecific variation. Evolution 66: 2697-2707.

Revell, L. J., D. L. Mahler, P. R. Peres-neto, and B. D. Redelings, 2011 A new phylogenetic method for identifying exceptional phenotypic diversification. Evolution 66: 135-146.

Rosenberg, N. A., and M. Nordborg, 2002 Genealogical trees, coalescent theory and the analysis of genetic polymorphisms. Nat. Rev. Genet. 3: 380-390.

Rousseau, C. M., M. G. Daniels, J. M. Carlson, C. Kadie, H. Crawford et al., 2008 HLA class I-driven evolution of human immunodeficiency virus type 1 subtype $C$ proteome: immune escape and viral load. J. Virol. 82: 6434-6446.

Sikorski, J., and E. Nevo, 2005 Adaptation and incipient sympatric speciation of Bacillus simplex under microclimatic contrast at "Evolution Canyons" I and II, Israel. Proc. Natl. Acad. Sci. USA 102: 15924-15929.

Stamatakis, A., 2006 RAxML-VI-HPC: maximum likelihood-based phylogenetic analyses with thousands of taxa and mixed models. Bioinformatics 22: 2688-2690.

Threlfall, E. J., 2000 Epidemic Salmonella typhimurium DT 104 a truly international multiresistant clone. J. Antimicrob. Chemother. 46: 7-10.

Uzzau, S., D. J. Brown, T. Wallis, S. Rubino, G. Leori et al., 2000 Host adapted serotypes of emphSalmonella enterica. Epidemiol. Infect. 125: 229-255.

Visscher, P. M., W. G. Hill, and N. R. Wray, 2008 Heritability in the genomics era concepts and misconceptions. Nat. Rev. Genet. 9: 255-266.

Yang, Z., and B. Rannala, 2012 Molecular phylogenetics: principles and practice. Nat. Rev. Genet. 13: 303-314.

Yang, Z., S. Kumar, and M. Nei, 1995 A new method of inference of ancestral nucleotide and amino acid sequences. Genetics 141: $1641-1650$.

Communicating editor: M. A. Beaumont 


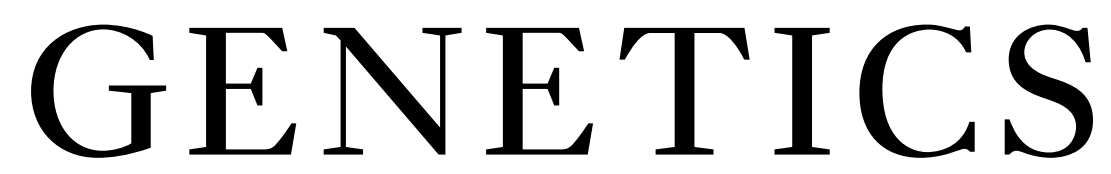

\section{Bayesian Inference of the Evolution of a Phenotype Distribution on a Phylogenetic Tree}

M. Azim Ansari and Xavier Didelot 


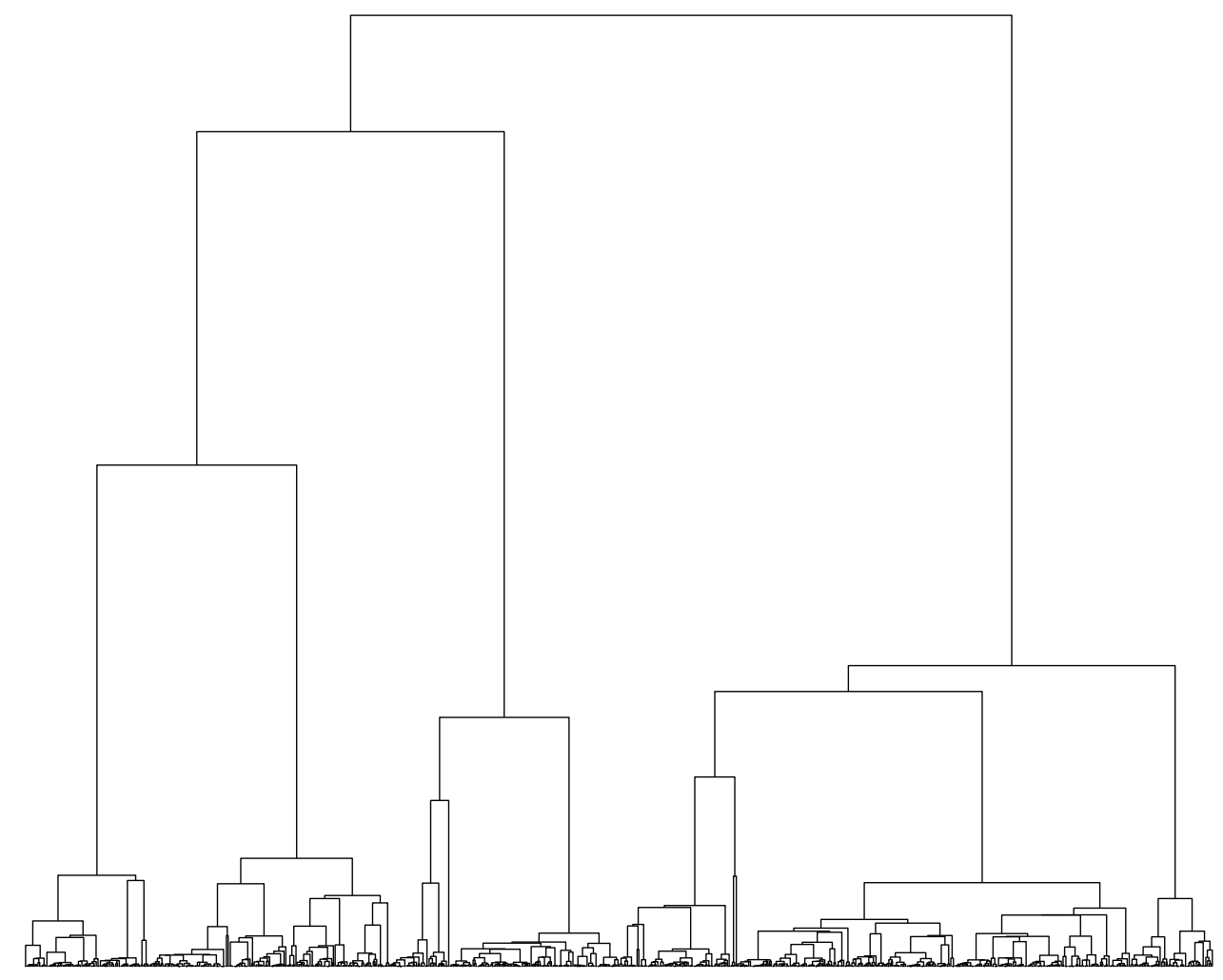

Figure S1: Tree used for the simulation studies. This tree was simulated using coalescent model with 1000 terminal branches. 
A

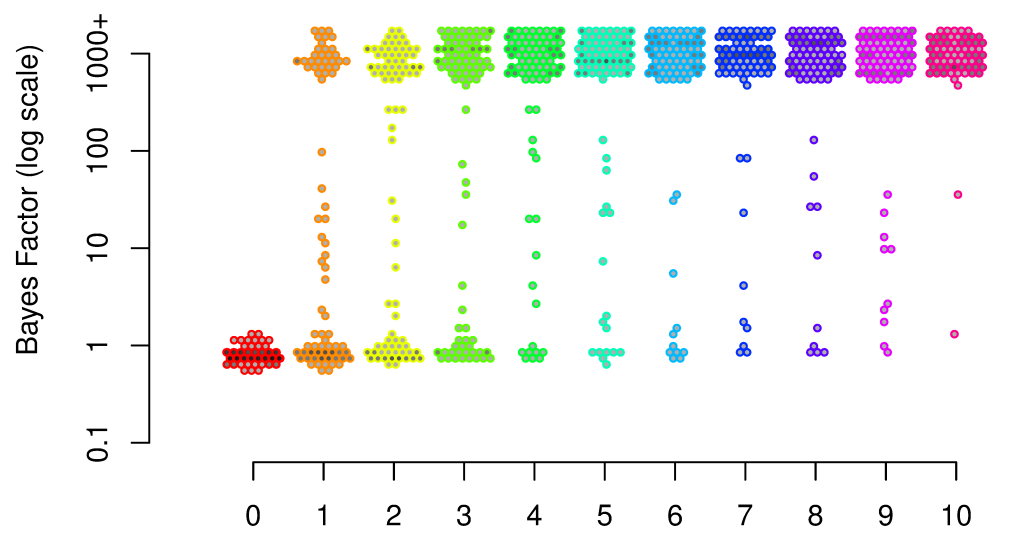

$\mathrm{B}$

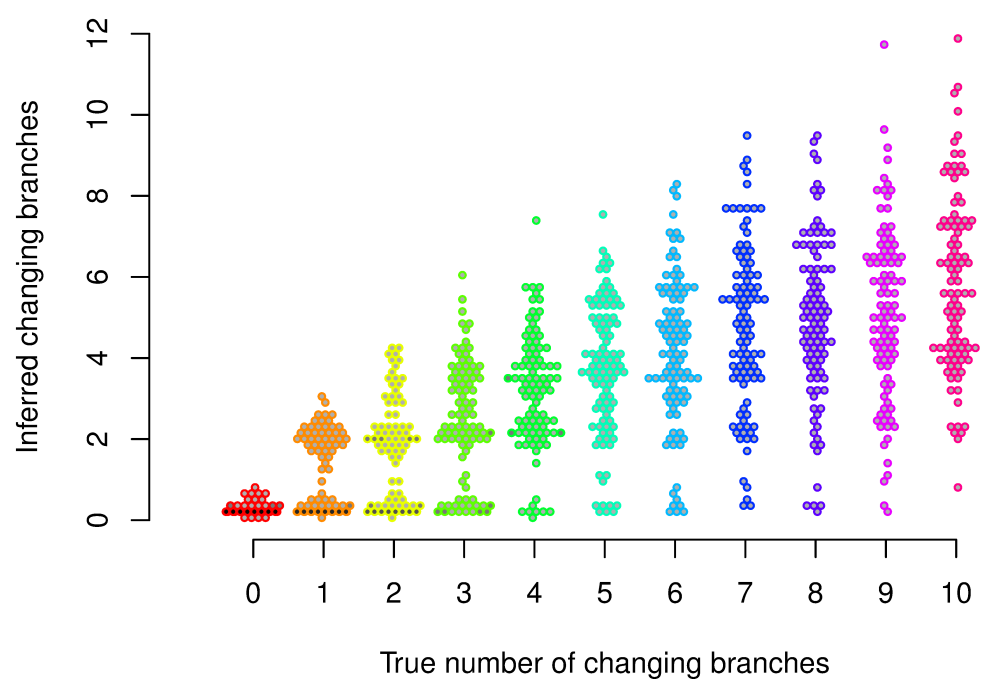

Figure S2: Equivalent of Figure 3 based on inference using a modified tree compared to the tree used for simulation. 


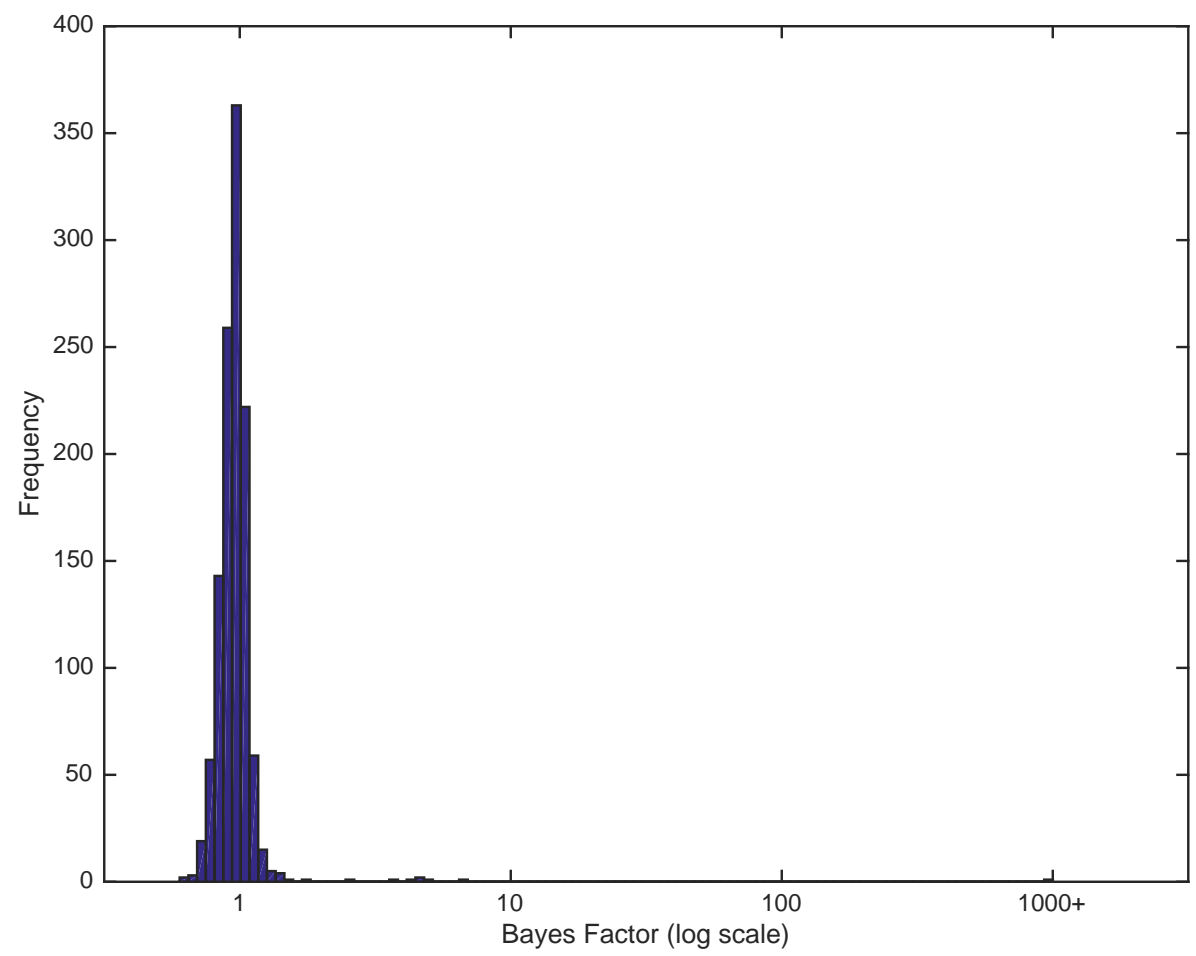

Figure S3: Bayes factor calculation for the HIV study. 


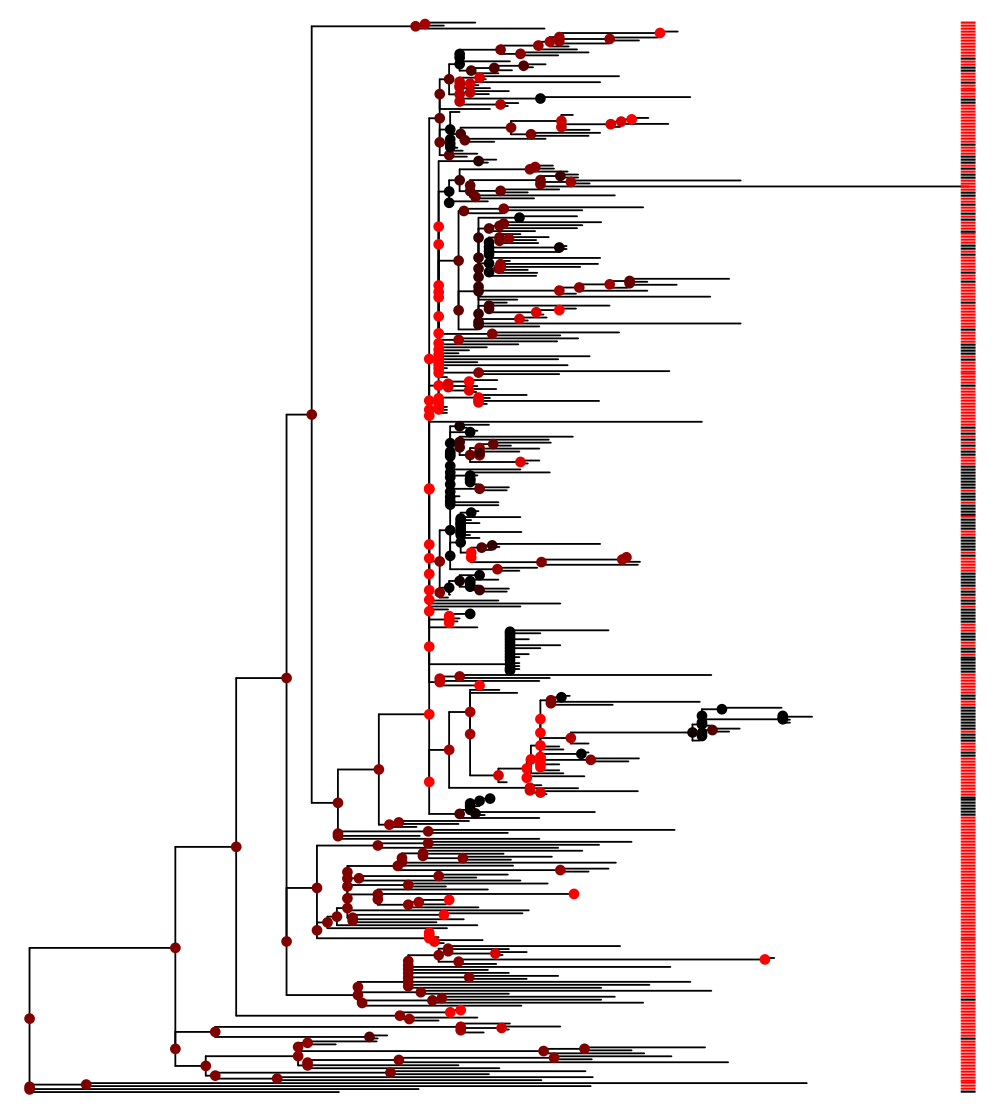

Figure S4: Application of ancestral state reconstruction to the Salmonella dataset. 


\section{File S1: Supplementary material}

\section{MCMC moves}

\section{Proposal for $b$}

Assuming that $\boldsymbol{b}^{\star}$ divides the tree into $n$ sections the Metropolis-Hastings ratio for this move is given by $h\left(\boldsymbol{b}, \boldsymbol{b}^{\star}\right)$ :

$$
\begin{aligned}
h\left(\boldsymbol{b}, \boldsymbol{b}^{\star}\right) & =1 \wedge \frac{(\Gamma(K))^{n} \prod_{i=1}^{n} \frac{\prod_{j=1}^{K} \Gamma\left(x_{i j}^{\star}+1\right)}{\Gamma\left(K+\sum_{j=1}^{K} x_{i j}^{\star}\right)} T e^{-T \lambda} \prod_{s=1}^{2 N-2}\left(e^{-\lambda l_{s}}\right)^{1-b_{s}^{\star}}\left(1-e^{-\lambda l_{s}}\right)^{b_{s}^{\star}}}{(\Gamma(K))^{m} \prod_{i=1}^{m} \frac{\prod_{j=1}^{K} \Gamma\left(x_{i j}+1\right)}{\Gamma\left(K+\sum_{j=1}^{K} x_{i j}\right)} T e^{-T \lambda} \prod_{s=1}^{2 N-2}\left(e^{-\lambda l_{s}}\right)^{1-b_{s}}\left(1-e^{-\lambda l_{s}}\right)^{b_{s}}} \\
& =1 \wedge \frac{(\Gamma(K))^{n-m} \prod_{i=1}^{n} \frac{\prod_{j=1}^{K} \Gamma\left(x_{i j}^{\star}+1\right)}{\Gamma\left(K+\sum_{j=1}^{K} x_{i j}^{\star}\right)} \prod_{s=1}^{2 N-2}\left(e^{-\lambda l_{s}}\right)^{1-b_{s}^{\star}}\left(1-e^{-\lambda l_{s}}\right)^{b_{s}^{\star}}}{\prod_{i=1}^{m} \frac{\prod_{j=1}^{K} \Gamma\left(x_{i j}+1\right)}{\Gamma\left(K+\sum_{j=1}^{K} x_{i j}\right)} \prod_{s=1}^{2 N-2}\left(e^{-\lambda l_{s}}\right)^{1-b_{s}}\left(1-e^{-\lambda l_{s}}\right)^{b_{s}}}
\end{aligned}
$$

Note that the prior on $\lambda$ cancelled out from the Metropolis-Hastings ratio. 


\section{Proposal for $\lambda$}

This proposal distribution is symmetric and therefore the Metropolis-Hastings ratio is given by:

$$
\begin{aligned}
h\left(\lambda, \lambda^{\star}\right) & =1 \wedge \frac{(\Gamma(K))^{m} \prod_{i=1}^{m} \frac{\prod_{j=1}^{K} \Gamma\left(x_{i j}+1\right)}{\Gamma\left(K+\sum_{j=1}^{K} x_{i j}\right)} T e^{-T \lambda^{\star}} \prod_{s=1}^{2 N-2}\left(e^{-\lambda^{\star} l_{s}}\right)^{1-b_{s}}\left(1-e^{-\lambda^{\star} l_{s}}\right)^{b_{s}}}{(\Gamma(K))^{m} \prod_{i=1}^{m} \frac{\prod_{j=1}^{K} \Gamma\left(x_{i j}+1\right)}{\Gamma\left(K+\sum_{j=1}^{K} x_{i j}\right)} T e^{-T \lambda} \prod_{s=1}^{2 N-2}\left(e^{-\lambda l_{s}}\right)^{1-b_{s}}\left(1-e^{-\lambda l_{s}}\right)^{b_{s}}} \\
& =1 \wedge \frac{e^{-T \lambda^{\star}} \prod_{s=1}^{2 N-2}\left(e^{-\lambda^{\star} l_{s}}\right)^{1-b_{s}}\left(1-e^{-\lambda^{\star} l_{s}}\right)^{b_{s}}}{e^{-T \lambda} \prod_{s=1}^{2 N-2}\left(e^{-\lambda l_{s}}\right)^{1-b_{s}}\left(1-e^{-\lambda l_{s}}\right)^{b_{s}}}
\end{aligned}
$$

